Division of Infectious Diseases,

University of California, Los Angeles

Twitter @tara_vijayan

Cite this as: $B M / 2022 ; 376: 0113$

http://dx.doi.org/10.1136/bmi.0113

Published: 14 January 2022

\title{
Raining in Los Angeles
}

\section{Amid climbing covid case numbers and with scarce resources, Tara Vijayan describes what it has been like to triage treatments that aim to prevent patients being hospitalised with covid-19}

\section{Tara Vijayan associate professor of medicine and medical director of antimicrobial stewardship}

It is 5am and pouring with rain in Los Angeles. I am wide awake.

I have grown used to the anxiety that the winter seasons now bring me. In December 2020, I started working on setting up our health system's programme to treat our highest risk covid-19 patients with monoclonal antibodies. These drugs had little data at the time to support their use to prevent hospitalisation from covid-19. ${ }^{1}$ With hundreds of patients already admitted to hospitals with covid-19 and the threat of running out of ICU beds edging closer, ${ }^{2}$ we grew desperate to stem the tide. Yet limited hospital space and treatment supply meant that we were only able to give these therapies to two patients a day.

We triaged dozens of referrals and would select from among those who were at the highest risk. We ensured that those who came from poorer communities who are disproportionately affected by this virus ${ }^{3}$ also had access to the treatment.

It felt like a drop in the bucket. But it was all we had. In those days, I was haunted by all the patients I had seen die in hospital over the year before and I was determined to do something. Anything.

More than once, colleagues begged me to treat their patients, sending me into a spiral of sadness and fear. I couldn't treat most of them. But I had to keep believing that treating two patients a day and trying to prevent them from going to the hospital was enough.

Eventually, we were able to treat more patients and it became clearer that the treatments worked. ${ }^{4}$ During a period of relative abundance, there was enough supply to treat the steady stream of high risk patients who had been infected with SARS-CoV-2. Our outpatient treatment team celebrated outdoors in May 2021 when LA had fewer patients with covid-19 and the number of referrals for treatment were down. We thought we had made it through the worst, and even the summer surge that followed was milder than the previous winter thanks to vaccines.

We started preparing for Omicron by the end of November. Early data suggested that only one monoclonal antibody treatment, sotrovimab, remained effective against this new variant and the supply was low. ${ }^{5}$ Even with emergency use authorisation from the US Food and Drug Administration for a new medication, tixagevimab/imdevimab (Evusheld), for pre-exposure prevention ${ }^{6}$ and another pill, nirmatrelvir/ritonavir (Paxlovid), for treatment, ${ }^{7}$ the supplies remained low. It was my job again to try to figure out how to administer these extremely scarce resources. Déjà vu. Back to treating a few patients a day.

The anxieties I have now are different. I have confidence in the vaccines, especially with the booster, which will prevent more people from going to the hospital than any treatment I can give them. Yet I remain worried for my patients who have not responded to the vaccination due to their lowered immune systems. I worry most for my patients who remain unvaccinated due to fear and mistrust. ${ }^{8}$

My team and I text back and forth. In one day, 45000 new people test positive for covid-19 in LA County. ${ }^{9}$ The hospital numbers are up. We continue triage for the few treatments we have available. But the rain keeps falling.

I receive a text from my 69 year old mother that shows a screenshot of her positive test. Two days before she had shared a meal with vaccinated friends at her local temple. I allow the stages of grief to pass through. Anger towards her for not being more careful. Guilt for not being able to help her as an infectious disease doctor. I know she will not be prioritised for treatment as she is fully vaccinated and not immunocompromised.

I recall the conversations I had with my mother in March 2020, soon after working a week in the hospital treating patients with covid-19. By that time, I had already witnessed the deaths. We both had heard of the ventilator scarcity in New York, of the tubes that were taken out of one patient and placed in another. We cried together as she expressed her wishes to me were she ever to get sick.

Things are different two years later and I know she will be okay with her three vaccine doses. I say this to patients exactly like her day after day. I know that in LA County the rate of hospitalisation for covid-19 is 28 times higher among those who are unvaccinated. ${ }^{10}$

In my final stage of grief, I lean on acceptance. I remember that her temple is her sanctuary and that she, like all of us, is exhausted by this pandemic. I allow us both a moment of grace.

I know I must reassure those patients who are at a lower risk of hospitalisation due to full vaccination. I know I must caution my colleagues to practise wisdom and not fear and refrain from trying to get their patients to "jump the line" for treatment in times of scarcity, and at the expense of equity.

And I know I must continue to reach out to those who choose to remain unvaccinated, particularly when they're from communities who have endured long histories of medical racism. ${ }^{11}$ Vaccines are a human 
right that I share with them. I wait patiently, visit after visit in my clinic, and tell these patients that while treatments remain limited, they will always have a place in line for the vaccines, rain or shine. Their trust in me is not a given but is earned.

As I drive home from work on the $405,{ }^{12}$ the rain falls hard in Los Angeles. But I know it will stop. And I know we can all allow ourselves some grace and still continue the work that is needed.

Competing interests: None declared.

Provenance and peer review: not commissioned, not peer reviewed.

1 McGinley L. Only one covid-19 treatment is designed to keep people out of the hospital. Many overburdened hospitals are not offering it. Washington Post. 31 December 2020.

https://www.washingtonpost.com/health/2020/12/31/covid-monoclonal-antibodies-unused/

2 Martinez L. Southern California Has Run Out Of ICU Beds For Coronavirus Patients. LAist. 17 December 2020. https://laist.com/news/icu-capacity-runs-out-socal-coronavirus-patients

3 Vijayan T, Shin M, Adamson PC, etal. Beyond the 405 and the 5: Geographic Variations and Factors Associated With Severe Acute Respiratory Syndrome Coronavirus 2 (SARS-CoV-2) Positivity Rates in Los Angeles County. Clin Infect Dis 2021;73:e2970-5. doi: 10.1093/cid/ciaa1692. pmid: 33141164

4 Webb B J, Buckel W, Vento T, et al. Real-world Effectiveness and Tolerability of Monoclonal Antibody Therapy for Ambulatory Patients With Early COVID-19, Open Forum Infectious Diseases, 2021 8;7:ofab331. doi: 10.1093/ofid/ofab331

5 Johnson CY. As the omicron variant threatens to wipe out monoclonal antibodies, the U.S. is saving up one that will still work. Washington Post. 16 December 2021. https://www.washingtonpost.com/health/2021/12/16/monoclonal-antibody-sotrovimab/

6 Coronavirus (COVID-19) Update: FDA Authorizes New Long-Acting Monoclonal Antibodies for Pre-exposure Prevention of COVID-19 in Certain Individuals. US Food and Drug Administration. 8 December 2021. https:/www.fda.gov/news-events/press-announcements/coronavirus-covid19-update-fda-authorizes-new-long-acting-monoclonal-antibodies-pre-exposure

7 Zimmer C, Robbins R. Pfizer's Covid Pill Works Well, Company Confirms in Final Analysis. New York Times. 14 December 2021. https://www.nytimes.com/2021/12/14/health/pfizer-covid-19pill-paxlovid.html

8 Batchlor E. I'm a Black Doctor. My Mom Still Won't Get Vaccinated. Atlantic. 1 September 2021 https://www.theatlantic.com/ideas/archive/2021/09/im-a-black-doctor-i-cant-persuade-my-momto-get-vaccinated/619933/.

9 Vega PLA. County sets another daily record with more than 45000 coronavirus cases. Los Angeles Times. 9 January 2022. https://www.latimes.com/california/story/2022-01-09/los-angelescounty-daily-record-45000-coronavirus-cases

10 LA County COVID data show stark difference between vaccinated and unvaccinated. ABC7.com 31 December 2021. https://abc7.com/covid-19-vaccine-los-angeles-county-coronavirus-dr-barbaraferrer-la-public-health/11408759/

11 Nuriddin A, Mooney G, White AIR. Reckoning with histories of medical racism and violence in the USA. Lancet 2020;396:949-51. doi: 10.1016/S0140-6736(20)32032-8. pmid: 33010829

12 Vijayan T. In the quiet of the holiday season, the City of Angels burns. BMJ Opinion. 5 January 2021. https://blogs.bmj.com/bmj/2021/01/05/tara-vijayan-in-the-quiet-of-the-holiday-seasonthe-city-of-angels-burns/ 